\title{
Optimizing self-pollinated crop breeding employing genomic selection: from schemes to updating training sets
}

Felipe Sabadin ( $\square$ felipe.sabadin@alumni.usp.br)

University of Sao Paulo: Universidade de Sao Paulo https://orcid.org/0000-0003-2937-1465

Julio César DoVale

Federal University of Ceara: Universidade Federal do Ceara

John Platten

IRRI: International Rice Research Institute

Roberto Fritsche-Neto

IRRI: International Rice Research Institute https://orcid.org/0000-0003-4310-0047

\section{Research Article}

Keywords: breeding scheme, Accuracy, sample size, response to selection

Posted Date: August 16th, 2021

DOI: https://doi.org/10.21203/rs.3.rs-805463/v1

License: (c) (i) This work is licensed under a Creative Commons Attribution 4.0 International License.

Read Full License 
5 Affiliations:

6 a Department of Genetics, "Luiz de Queiroz" College of Agriculture, University of São Paulo, Brazil.

$7 \quad{ }^{\mathrm{b}}$ Department of Crop Science, Federal University of Ceará, Fortaleza, Ceará, Brazil.

$8 \quad{ }^{\mathrm{c}}$ International Rice Research Institute (IRRI), Los Baños, Philippines.

9

$10 *$ Corresponding author

11 Roberto Fritsche-Neto r.fritscheneto@irri.org

12

13 ORCID of the authors:

14 Roberto Fritsche-Neto (RFN): 0000-0003-4310-0047

15 Felipe Sabadin (FS): 0000-0003-2937-1465

16 Julio César DoVale (JCD): 0000-0002-3497-9793

17 John Platten (JP): 0000-0001-6137-5955 


\section{ABSTRACT}

21 Long-term breeding schemes employing genomic selection (GS) can boost the response to selection per year. Although 22 several studies show that GS delivers a higher response to selection, only a few analyze the best strategy to employ it, 23 specifically how often and in what manner the training set (TS) should be updated. Therefore, we used stochastic simulation 24 to compare in a long-term breeding program of a hypothetical self-pollinated crop five different strategies to implement GS 25 in the line fixation stage and four methods and sizes to update the TS. Moreover, among breeding schemes, we proposed a 26 new approach for using GS to select the best individuals in each F2 progeny based on genomic estimated breeding and 27 divergence and crossed them to generate a new recombination event. Finally, we compared these schemes to the traditional 28 phenotypic selection and drift. Our results showed that using GS in F2 followed by the phenotypic selection of new parentals 29 in F4 was the best scenario. Furthermore, adding a new set of training data every cycle (over 800) to update the TS maintains 30 the accuracy at satisfactory levels for many more generations, showing that more data is better than optimizing the genetic 31 relationship between TS and the targeted population in a closed system. Hence, we believe that these results may help breeders 32 optimize GS in their programs and improve genetic gain in long-term schemes.

34 Keywords: breeding scheme; Accuracy; sample size; response to selection; 
DECLARATIONS

36

37 Funding: AGGRi Alliance (Accelerated Genetic Gain in Rice in South Asia and Africa - OPP1194889) - Bill and Melinda 38 Gates Foundation (BMGF).

39

40 Conflicts of Interest: The authors declare no conflict of interest.

41

42 Availability of data: all the data is available as supplementary information.

43

44 Code availability: all the scripts are available as supplementary information.

45

46 Ethical standards: Not applicable

47

48 AUTHOR CONTRIBUTION STATEMENT

49 FS wrote the manuscript, discussion, contributing to ideas and graphs. JCD and JP contributed to the writing, mainly 50 discussion. RFN elaborated on the hypothesis, conducted the analyses, interpreted the results, and contributed to the writing.

51 All authors read and approved the final manuscript.

52

\section{ACKNOWLEDGMENTS}

54 To Dr. Gary Atlin (Bill and Melinda Gates Foundation), IRRI Breeders, Excellence in Breeding - CGIAR platform, and 55 Allogamous Breeding lab-USP members for the comments and suggestions.

\section{KEY MESSAGE}

58 Update the training set every cycle is a key factor in GS accuracy. 
61

62

Genomic selection (GS) has successfully been employed in plant and animal breeding (Heffner et al. 2011a; Spindel et al. 2015; Wolc et al. 2015; Garner et al. 2016). In this technique, breeders build a training set (TS) containing genotyped and phenotyped individuals to estimate the marker effects and use them to calculate genomic estimated breeding values (GEBV) of only genotyped individuals (Meuwissen et al. 2001). Since the selection of individuals does not need their phenotypic information, these genotypes can be selected early, which permits faster breeding schemes and increases the genetic gain (Beyene et al. 2015; Voss-Fels et al. 2019). However, several parameters influence the accuracy of GEBV prediction, including TS size (Heffner et al. 2011b; Lorenz 2013), population structure (Guo et al. 2014), the genetic relationship between the TS and selection candidate genotypes (Lorenz and Smith 2015), and trait heritability (Combs and Bernardo 2013).

Several studies have examined optimal employment of GS on self-pollinated crop breeding program (Lorenz et al. 2012; He et al. 2016; Michel et al. 2016). The self-pollinated crop breeding programs are composed of three phases: create genetic variation, identify desirable recombinant lines within progenies, and stabilize and advance the desired genotype (Brown et al. 2014). There are several breeding methods to increase the homozygosity during the line fixation step, and single seed descent (SSD) is the method most suited to accelerating a breeding scheme. In rice, especially in the IRRI breeding program, the rapid generation advanced (RGA) method, which consists of an SSD method conducted in greenhouses or screenhouses, is employed to accelerate and shorten the growth cycle (Collard et al. 2017). The combination between GS and the RGA can boost genetic gain in rice long-term breeding schemes principally due to shortening each cycle, which increases the response to selection (Falconer and Mackay 1996).

Long-term breeding schemes should be analyzed concerning different genetic parameters, as genetic gain, response to selection, genetic variance, and prediction accuracy to determine the best long-term strategy for maximizing results. Even though there are several studies involving GS in long-term recurrent selection schemes (Goddard 2009; Jannink 2010; Muleta et al. 2019), only a few test the best stage to apply it (Bassi et al. 2016; Gaynor et al. 2017). Mendonça et al. (2020) showed that GS increases the efficiency in advanced phases in a soybean breeding program using a low-intensity selection for quantitative traits in the early stages. Marulanda et al. (2016) verified different strategies to apply GS in hybrid breeding programs in several self-pollinated crops. They concluded that using GS in stage 1 followed by a final phenotypic selection stage produced the highest genetic progress. Hence, there is room to optimize the strategy and stage for using GS to boost genetic gain and improve efficiency in a breeding program. 
90 cycles (Jannink 2010; Muleta et al. 2019). This phenomenon might be due to the decrease in genetic relationship between

91 TS and selection candidate genotypes (Lorenz and Smith 2015), the breakdown of LD between marker and QTL due to recombination (Jannink 2010; Müller et al. 2017), and the lack of genetic variability of the population. In this context, a few

93 studies claim the requirement of updating the TS (Jannink 2010; Muleta et al. 2019; DoVale et al. 2021) to reduce the accuracy

94 decline across breeding cycles. For instance, Neyhart et al. (2017) used different methods to select individuals to add into the

95 TS during recurrent selection cycles. They concluded that a better strategy to decrease accuracy decline is selecting 96 individuals genetically most related to selection candidate genotypes. Therefore, our study aimed to optimize the use of GS 97 and identify a method to update the training set to achieve that brings greater efficiency in self-pollinated crop breeding 98 programs. For that, we used stochastic simulation to compare five different strategies to implement GS in the line fixation 99 stage and four methods and sizes to update TS in a long-term breeding program of a hypothetical self-pollinated crop.

100 Moreover, among breeding schemes, we proposed a new method for using GS to select the best individuals in each F2 progeny

101 based on genomic estimated breeding and divergence and crossed them to generate a new recombination event. Finally, we 102 compared these schemes to the traditional phenotypic selection and drift. 
In our study, we compared different GS strategies applied in a long-term breeding program. For that, we used rice (Oryza sativa L.) as a model self-pollinated crop and stochastic simulations performed by the AlphaSimR package (Gaynor et al. 2021). Furthermore, we evaluated different strategies for updating the TS and how it influences genetic parameters over breeding cycles.

\section{Long-term breeding schemes employing genomic selection}

Historical population and genetic parameters

The historical rice founder population was simulated as 1,000 unique diploid inbred individuals, with 12 chromosome pairs each using a Markovian Coalescent Simulator (MaCS) (Chen et al. 2009). 1,644 biallelic segregating sites were considered, uniformly distributed across chromosomes and 360 segregating loci randomly sampled as quantitative trait nucleotides (QTN) and 994 segregating loci as single-nucleotide polymorphism (SNP).

The target of simulation was a quantitative trait, such as yield. The genetic parameters obtained by Li et al. (2008) were used. Each QTN received randomly-allocated additive and dominance effects. Genetic values for each genotype were obtained by summing all additive and dominance effects for all QTN. Additive effects $(a)$ were sampled of a gamma distribution with scale and shape parameters equal to 1 and randomly assigned for each QTN. Similarly, dominance effects

(d) for each QTN were computed by multiplying the absolute value of its additive effect $\left(a_{i}\right)$ by locus-specific dominance degree $\left(\delta_{i}\right)$. Dominance degrees were sampled of a Gaussian distribution with $\delta_{i} \sim N\left(\mu_{\delta}, \sigma_{\delta}^{2}\right)$, where $\mu_{\delta}$ is the average dominance degree equal to 0.22 and $\sigma_{\delta}^{2}$ is the dominance variance equal to 0.50 . Dominance effects were assigned for each QTN according to the equation below:

$$
d_{i}=\left\{\begin{aligned}
0, & \text { if QTN is homozygous } \\
\delta_{i} \times\left|a_{i}\right|, & \text { if QTN is heterozygous }
\end{aligned}\right.
$$



steps were conducted independently. Parental lines (24 or 48) were randomly sampled based on their phenotypic value to build the base population. As a starting point to consider a program representative of current 4-year rice breeding programs, five traditional recurrent selection cycles were simulated totaling 20 years of the program. First, the parental lines were randomly crossed to generate $30 \mathrm{~F}_{1}$ plants, which were selfed to produce $230 \mathrm{~F}_{2}$ plants from each cross (Cobb et al. 2019). SSD conducted the line fixation stage until the $\mathrm{F}_{6}$, where the best individuals based on their phenotypic values were selected to found the next breeding cycle. After three recurrent breeding cycles, we obtained the initial population to evaluate the genomic recurrent selection breeding schemes.

The base TS was composed of 1,530 inbreed lines originated by crossing between individuals from the historical population. Markers effects were predicted using the ridge-regression best linear unbiased prediction (RRBLUP) (Endelman 2011) according to the equation below:

$$
y=1 \mu+Z_{u} u+\varepsilon
$$

where $\boldsymbol{y}$ is the vector of individual phenotypic values from the TS; $\mu$ is the mean (intercept); $\boldsymbol{u}$ is the vector of marker effects, where $\boldsymbol{u} \sim N\left(\mathbf{0}, \boldsymbol{I} \sigma_{u}^{2}\right)$; and $\boldsymbol{\varepsilon}$ is the vector of random residuals. $\mathbf{1}$ is the vector of ones and $\boldsymbol{Z}_{\boldsymbol{u}}$ is the incidence matrix of TS genotypes for $m$ markers. $\boldsymbol{Z}_{\boldsymbol{u}}$ is coded as 1 for homozygous $\mathrm{A}_{1} \mathrm{~A}_{1},-1$ for homozygous $\mathrm{A}_{2} \mathrm{~A}_{2}$, and 0 for heterozygous $\mathrm{A}_{1} \mathrm{~A}_{2}$.

To perform the GS, the genomic estimated breeding value (GEBV) were estimated using the following equation:

$\boldsymbol{G E} \boldsymbol{B} \boldsymbol{V}=\boldsymbol{M u}$, where $\boldsymbol{M}$ is the incidence matrix of selection candidate genotypes, and $\boldsymbol{u}$ is the vector of predicted marker effects.

Breeding schemes simulations

Simulations were based on the rice breeding program structure from the International Rice Research Institute (IRRI) (Collard et al., 2019) as a representative self-pollinated crop. For all scenarios, the line fixation phase was conducted by the single-seed descent (SSD) method, which consists of collecting one seed from each segregating plant to advance for the next stage until it reaches a high homozygosity level. Seven breeding schemes were compared, with different timelines and GS 
162

163

164

165

166

167

168

169

170

171

172

173

174

175

176

177

178

179

180

181

182

183

184

185

186

187

188

189

190

procedures (Fig.1). Five schemes used GS in distinct stages during the line fixation phase, and two phenotypic breeding schemes (traditional and drift) were used as baselines. Furthermore, two group sizes of parental lines (24 and 48) were analyzed to populate the crossing block, totaling 14 different scenarios. In this case, we make a naïve assumption, which the number of parents is a proxy to the effective population size $(\mathrm{Ne})$.

In the first cycle, from crossing to $\mathrm{F}_{2}$ generation were the same for all breeding schemes. Parental lines were crossed to generate 30 random crosses between them. Then, $F_{1}$ plants were selfed to produce a $F_{2}$ segregating generation with $230 \mathrm{~F}_{2}$ plants per cross (Cobb et al. 2019). From the $F_{2}$ phase, the different long-term breeding schemes were applied until the new parental group selection for the next cycle. After the second cycle, each scheme followed independently and simulated over 20 breeding cycles and 40 replicates, totaling 800 estimates (Fig.1).

Fig. 1 Scheme of the first cycle of the long-term breeding schemes. In the final of each scheme, a parental group was selected for recycling and compound the next cycle that works independently

Traditional (Trad) and drift (Drift) schemes were conducted by the SSD method from F2 to F6 generation. Each generation lasted about 180 days, totaling four years for each cycle. These two schemes differed on new parental group selection, where they were selected based on the best phenotypic values (Trad) and a random sample (Drift). For breeding schemes with GS, the line fixation phase was conducted by the rapid generation advanced (RGA) method. The RGA method is an SSD method carried out in greenhouses or screenhouses to accelerate and shorten the growth cycle ( 90-100 days). Collard et al. (2017) present a good review of the RGA method. Below are details about each breeding scheme, along with how GS was applied:

- $\mathrm{GS}_{\mathrm{F} 2}$ : GS was performed in the $\mathrm{F}_{2}$ stage, where all plants were genotyped. Individuals with the best GEBV in $\mathrm{F}_{2}$ were selected (24 or 48), regardless of progenies, to compound the next cycle (one year per cycle).

- $\mathrm{GS}_{\mathrm{F} 2} \mathrm{~S}_{\mathrm{F} 4}$ : GS was performed in the $\mathrm{F}_{2}$ stage, and genotypes with the best GEBV within each progeny were selected for the next generation $\left(\mathrm{F}_{3}\right)$. So, $230 \mathrm{~F}_{3}$ plants from the selected $\mathrm{F}_{2}$ plant were conducted until $\mathrm{F}_{4}$. In $\mathrm{F}_{4}$, individuals with the best phenotypic value were selected, regardless of progenies, to compound the next cycle (2 years per cycle).

- $\mathrm{GS}_{\mathrm{F} 4}$ : GS was performed in the $\mathrm{F}_{4}$ stage, where all plants were genotyped. Individuals with the best GEBV in $\mathrm{F}_{4}$ were selected ( 24 or 48 ), regardless of progenies, to compound the next cycle ( 2 years per cycle). 
- GS_FSb: GS was performed in the $F_{2}$ stage, and the two individuals with the best GEBV within each progeny were selected and crossed to generate $230 \mathrm{~F}_{1 \text { seg }}$ segregating plants. These plants were conducted until $\mathrm{F}_{4}$, and individuals with the best phenotypic value were selected, regardless of progenies, to compound the next cycle (2.33 years per cycle).

- GS_FSd: GS was performed in the $\mathrm{F}_{2}$ stage, and the best individuals (10\%) based on GEBV were selected to calculate the Euclidean genetic distance between them. Within each progeny, the most divergent sibs were crossed to generate $230 \mathrm{~F}_{1 \text { seg }}$ segregating plants. These plants were conducted until $\mathrm{F}_{4}$, and individuals with the best phenotypic value were selected, regardless of progenies, to compound the next cycle (2.33 years per cycle).

The average genetic value, genetic value of the best genotype, additive genetic variance, and prediction accuracy were calculated for each breeding cycle. The prediction accuracy was calculated as a Pearson correlation between true genetic values and GEBV. For Trad and Drift schemes, the prediction accuracy was computed as the square root of heritability $\left(h^{2}\right)$. Furthermore, the response to selection was calculated following the equation:

$$
R S=\frac{X_{i}-X_{0}}{t}
$$

where $R S$ is the relative response to selection to the previous cycle; $X_{i}$ is the genetic value mean of the parental group in the $i$ cycle; $X_{0}$ is the genetic value mean of the previous parental group; and $t$ is the breeding cycle time.

\section{Training sets update scenarios}

The influence of updating the TS on long-term GS breeding schemes was examined by tested different strategies to update this. The crossing block was fixed at 48 parental lines for each cycle and the $\mathrm{GS}_{\mathrm{F} 2} \mathrm{~S}_{\mathrm{F} 4}$ scheme used as the breeding strategy. As benchmark strategies, we used Trad and Drift schemes. Below are details about the updating scenarios:

- $\quad \mathrm{TSC}_{0}$ : the TS was composed of 1,530 individuals. It was built just one time and used for all cycles, without updates. Markers effects were used to predicted selection candidate genotypes in the future cycles.

- $\mathrm{TSC}_{\mathrm{N}}$ : TS was initially composed of 390 individuals. Each new cycle, a new TS was built with 13 random individuals within each $\mathrm{F}_{4}$ progeny, totaling 390 individuals. Marker effects were estimated in each cycle and used to predicted selection candidate genotypes in the next cycle. 
- $\mathrm{TS}_{\mathrm{ALL}}$ : TS was initially composed of 390 individuals. In every cycle, a new group was added consisting of 390 individuals ( 13 random individuals within each $\mathrm{F}_{4}$ progeny). The TS thus increased by 390 individuals each cycle $(390,780,1170, \ldots, 3,900)$. Marker effects were estimated in each cycle and used to predicted selection candidate genotypes in the next cycle.

- $\mathrm{TS}_{\mathrm{GPO}}$ : TS was composed of three sets of 390 individuals, one set per generation (grandparents, parents, and offspring). Every cycle, the newest set, selecting 13 randomly individuals within each $\mathrm{F}_{4}$ progeny totaling 390 individuals, was included and the oldest one removed. Markers effects were estimated in each cycle and used to predicted selection candidate genotypes in the next cycle. the TS every breeding cycle (individuals phenotyped and genotyped), aiming to recalibrate the marker effects and keep the GS accuracy at satisfactory levels for more generations. and response to selection were calculated for each cycle. 


\section{Influence of the breeding schemes and number of parental lines}

The start point (intercept) of 24 and 48 parental lines was equal as the same burn-in was used for both (48). Then, each parental group size was conducted independently in the further scenarios (Fig.2). In this context, GS-based methods showed similar performances regardless of the number of parents, concerning population mean (genetic mean), the best genotype performance, accuracy, and additive genetic variability (Fig.2a and 2b). Conversely, using 24 parents, the population plateau was reached earlier for the traditional SSD than using 48 parents. GS_FSb and $\mathrm{GS}_{\mathrm{F} 2} \mathrm{~S}_{\mathrm{F} 4}$ were the best Gsbreeding cycles (Fig.2).

Our results revealed that additive variance reduced over the breeding cycles for all breeding schemes regardless of the number of parental lines (Fig.2c). Also, using 48 parental lines showed a slightly higher value of additive variance over the cycles than 24 parental lines. Furthermore, the drift effect on the genetic variability was much more pronounced in 24 parents, which can vanish the genetic variability and genetic gains over the cycles (Fig.2c). On the other hand, all GS breeding schemes revealed a reduction of accuracy across the breeding cycles (Fig.2d). Indeed, accuracy values in GS schemes displayed negative values from the eighth cycle for 24 parentals, though for 48 parentals, only GS_FSd and GS_FSb showed negative values from the eighth and tenth cycle, respectively.

Fig. 2 Population mean (a), the best genotype (b), additive variance (c), and accuracy of selection (d) over 20 long-term breeding schemes using two sizes of parental lines ( $\mathrm{N}=24$ and $\mathrm{N}=48)$. Each colored line represents a breeding scheme.

Concerning the response to selection after a defined period (ten years) of breeding, which is much more realistic because each method has a different length, the $\mathrm{GS}_{\mathrm{F} 2} \mathrm{~S}_{\mathrm{F} 4}$ method outperformed the others, and specifically, the traditional SSD the advantage of using that method was higher than $93 \%$ (Fig.3). $\mathrm{GS}_{\mathrm{F} 2} \mathrm{~S}_{\mathrm{F} 4}$ outperformed all the others among the GS methods, being $44 \%$ better than fast recycling methods, such as $\mathrm{GS}_{\mathrm{F} 2}$, highlighting the trade-off between the number of cycles 
perspective, perhaps reflects stochastic variation or does not compensate for the risks due to the drift. Therefore, for further comparisons, we considered 48 parents and the $\mathrm{GS}_{\mathrm{F} 2} \mathrm{~S}_{\mathrm{F} 4}$ breeding scheme.

Fig. 3 Genetic gains after ten years of breeding using different selection schemes and two sizes of parental lines ( $N=24$ and $\mathrm{N}=48$.

\section{Effect of training set update}

After the breeding scheme evaluation, different ways to update the TS were compared, re-estimating the marker effects, to keep GS performances satisfactory in long-term breeding schemes. Overall, the updating methods $\left(\mathrm{TSC}_{\mathrm{N}}, \mathrm{TS}_{\mathrm{ALL}}\right.$, and $\mathrm{TS}_{\mathrm{GPO}}$ ) produced similar responses in terms of accuracy over cycles and outperformed the $\mathrm{TS}_{0}$ (no updates), which presented a steep decline after just two to four breeding cycles (Fig.4). Differences negligible among the updated scenarios, so $\mathrm{TS}_{\mathrm{ALL}}$ was considered the best due to ease of implementation and a more stable trend over cycles. Also, if for any reason

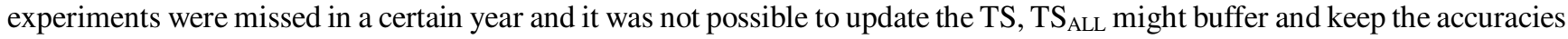
at higher levels due to its size. Therefore, for the final comparisons, the latter scenario for updating TS was considered.

Fig. 4 Accuracy of selection over 20 breeding cycles via $\mathrm{GS}_{\mathrm{F} 2} \mathrm{~S}_{\mathrm{F} 4}$ scheme considering 48 parental lines using different methods to update the training set. Each colored line represents an updating training set method.

Finally, we compared different sample sizes to update the TS every breeding cycle. Overall, by adding sample sizes of 800 ( $10 \%$ of the breeding program the total breeding program population size after RGA) or more every cycle it is possible to keep the GS accuracy at satisfactory levels for more cycles (up 8 or 10), outperforming the $\mathrm{TS}_{0}$ (no updates) or small sample sizes, such as 390 (Fig.5). After 8 or 10 cycles, there is a steady decay even for the best methods, which might be due to the lack of additive genetic variability in the population (Fig.2c).

Fig. 5 Accuracy of selection over 20 breeding cycles via $\mathrm{GS}_{\mathrm{F} 2} \mathrm{~S}_{\mathrm{F} 4}$ scheme considering 48 parental lines, the $\mathrm{TS}_{\mathrm{ALL}}$ method, and different sample sizes to update every cycle's training set. Each colored line represents an updating training sample size. 


\section{Breeding schemes and number of parental lines}

GS can speed up breeding schemes and increase genetic gain (Jannink et al. 2010; Varshney et al. 2017). However,

to maximize the breeding program efficiency, it is essential to decide on how and at which stage to apply GS. In this study,

different long-term breeding strategies for implemented GS were tested based on a simulated rice breeding program.

Furthermore, a new approach is proposed for using GS during the line fixation stage. In this approach, two individuals (best

ones - GS_FSb, and most divergent - GS_FSd) within each $\mathrm{F}_{2}$ progeny are identified and crossed to generate a new recombination event. These progenies are then fixed using the RGA method until $\mathrm{F}_{4}$ to select new parents for the next recurrent cycle.

Firstly, these results showed that the number of parental lines to compose the crossing block influences all genetic parameters over breeding cycles. This influence was expected due to the relationship between the number of parental lines with the effective population size (Ne) and the selection intensity. In the situations examined here, the number of crosses (crosses $=30$ ) was the same in both scenarios ( 24 and 48 parentals). However, a lower number of parents increases the number of crosses with the same parental, which reduces the final Ne, and consequently, the genetic variance. Also, a smaller $\mathrm{Ne}$ increases the effect caused by the drift (Hartl and Clark 2006), which brings a reduction in genetic variance in long-term breeding cycles. The Drift scheme acted as one of the benchmarks for this purpose. In the Drift scheme, using 24 parentals showed an additive genetic variance reduction over breeding cycles, whereas using 48 parental lines showed higher resilience to this effect. Furthermore, the Ne is a crucial factor for breeders as programs with a smaller Ne will become inbred faster and show no further response to selection (Cobb et al. 2019).

Several studies report the increase of response to selection in breeding schemes that use GS (Gorjanc et al. 2018; Muleta et al. 2019). In this study, long-term breeding schemes including GS showed values between $200 \%\left(\mathrm{GS}_{\mathrm{F} 2}\right)$ and $608 \%$ (GS_FSd) higher than phenotypic selection (Trad) in the first breeding cycle for response to selection per year (Fig.2c). This increase was principally due to the shortening of the breeding cycle with the implementation of GS. However, as the breeding cycles progress, they showed a rapid decrease in response to selection. Long-term breeding schemes with GS have been observed to exhaust the genetic variance faster than phenotypic selection because of the tendency of GS to select closelyrelated individuals, thereby accelerating inbreeding (Jannink 2010). These results also show a greater decline in genetic 
322

323

324

325

326

327

328

329

330

331

332

333

334

335

336

337

338

339

340

341

342

343

344

345

346

347

348

349

350

decays faster (Fig.2). However, this effect can be mitigated and offset by preserving a larger $\mathrm{Ne}$ (48 parent scenario), reducing the genetic variance consuming by GS over breeding cycles and permitting long-term genetic gain.

Genomic selection also increases genetic gain through shortening the breeding cycle, since phenotypic information is not required to select the best genotypes (Jannink et al. 2010; Lorenz et al. 2011; Crossa et al. 2017). Thus, the breeder can use methods to shrink the growth cycle and increase generations per year. Nevertheless, to shorten the growth cycle, the mode of reproduction of the species associated with the breeding method must be considered to reach the desirable effect (Brown et al. 2014). Rice does not currently have scalable and feasible procedures to generate doubled-haploid populations, so in this study the RGA method was used to advance generations and increase endogamy in our GS breeding schemes. This method reduces the rice growth cycle to 90-100 days, depending on the genotype (Collard et al. 2017). Differences in the number of generations in the breeding schemes examined led to differences in the total cycle time, with the Trad scheme taking four years to complete each cycle, whereas the $\mathrm{GS}_{\mathrm{F} 2}$ lasted only six months. The $\mathrm{GS}_{\mathrm{F} 2} \mathrm{~S}_{4}$ was observed to yield $93 \%$ higher genetics gain than the Trad scheme and $44 \%$ better than $\mathrm{GS}_{\mathrm{F} 2}$ in terms of genetic gains in ten years of breeding (Fig.3). Another interesting point is the best scenarios are composed of 2-stages selection, whereas all others are 1-stage or combined. This result shows that besides longer, 2-stages, combining GS and phenotypic selection, compensate rather than apply rapid cycling, using only GS at the stage-1 for recycling parents. Therefore, our results reinforce that to obtain sustainable longterm genetic gain in long-term breeding schemes is crucial to consider a balance between genetic gain, accuracy, time, and genetic variance over the breeding cycles (Jannink et al. 2010). In this context, some questions arise for further studies: do we get progressive increases in effectiveness with 3, 4, 5 stages of selection? E.g., GS at F2 + GS at F4 + Phenotypic at F6? Do we need different types of selection? GS and phenotypic are used here (2 different types). Would the same response be shown with, e.g., two rounds of GS?

Few studies explore the best phase or stage to apply GS to maximize the genetic gain in long-term breeding schemes (Gorjanc et al.; Bassi et al. 2016). A new method was examined here employing GS in the line fixation stage in a rice breeding program. In this scheme, a cross was simulated between two individuals selected by GS in the $\mathrm{F}_{2}$ generation (GS_FSb and GS_FSd) considering the best GEBVs (GS_FSb) and most divergent between best GEBVs (GS_FSd). Among the GS-based schemes, the GS_FSb reached the highest population mean over the breeding cycles (Fig.2a). The strategy of crossing the best individuals based on GEBV probably enriched the parents in which major-effect alleles were fixed since these individuals may have the same genotype for these alleles (Zhong et al. 2009; Jannink 2010). Also, loci with minor effects, but important for the trait, could be divergent between these individuals, and recombination between these loci may increase the probability of a future line with this favorable allele in homozygosity. On the other hand, when we crossed the most divergent individuals, 
351

352

353

354

355

356

357

358

359

360

361

362

363

364

365

366

367

368

369

370

371

372

373

374

375

376

377

378

major loci may have different alleles in each genotype. The probability of achieving a future line with the favorable allele in this locus is therefore lower, and indeed GS_FSd had one of the worst results considering genetic value over breeding cycles.

Even though GS_FSb has shown the highest population mean over breeding cycles, its values were close to the $\mathrm{GS}_{\mathrm{F} 2} \mathrm{~S}_{\mathrm{F} 4}$ scheme. However, further considering genetic gain, it is crucial to regard the practicality to realize each breeding scheme in real conditions. Making a new cross between two individuals from an $\mathrm{F}_{2}$ progeny brings additional labor and costs. Hence, since the difference between GS_FSb and $\mathrm{GS}_{\mathrm{F} 2} \mathrm{~S}_{\mathrm{F} 4}$ is negligible, we consider the $\mathrm{GS}_{\mathrm{F} 2} \mathrm{~S}_{\mathrm{F} 4}$ the best scenario to employ GS.

\section{Effect of training set update strategies}

The second objective of this study was to identify a better strategy to update the TS to guarantee a higher genetic gain and avoid the decline of accuracy across long-term breeding cycles. As mentioned, all genomic breeding schemes showed a reduction of accuracy over breeding cycles (Fig.2d). After the first breeding cycle, the prediction accuracy showed a substantial decline, and by the last breeding cycles GS was practically random, with the accuracy was close to zero. This situation can hinder the selection of the best genotypes, and consequently, the genetic mean of the population does not increase and may sometimes decrease (Jannink 2010; Neyhart et al. 2017). This observation is consistent with several other studies showing the reduction of accuracy of genomic prediction in long-term breeding schemes (Jannink 2010; Müller et al. 2017; Muleta et al. 2019). Working with GS optimization in a sorghum breeding program, Muleta et al. (2019) verified that the prediction accuracy declined over breeding cycles, especially for oligogenic traits. The latter study also showed that updating the TS slowed the decline in accuracy over breeding cycles but did not prevent this entirely. In these results, all scenarios involving GS with TS update reached the best performance for population mean compared to phenotypic selection, at least until the sixth breeding cycle. In initial cycles, $\mathrm{TSC}_{0}$ had the highest population mean values, which shows that a larger TS delivers a higher accuracy (Cericola et al. 2017; Norman et al. 2018) because a larger number of allelic observations is required to accurately predict a small effect QTL (Gilmour 2007). However, the $\mathrm{TSC}_{0}$ displayed a greater decrease in accuracy across breeding cycles since that this scenario did not update the TS (Fig.3). This accuracy reduction may promote a lower population mean than other updating scenarios after the fourth cycle. Amongst scenarios that did include updating of the TS, the $\mathrm{TSC}_{\mathrm{N}}$ scenario was unstable and slightly worse than $\mathrm{TS}_{\mathrm{GPO}}$ or $\mathrm{TS}_{\mathrm{ALL}}$. These results show that it is crucial to update the $\mathrm{TS}$ keeping high the genetic relationship between the TS and selection candidate genotypes. 
Another question about updating the TS is how and how many to select the individuals to add them to this. Neyhart et al. (2017) studied different updating TS methods in a barley long-term breeding program. Updating the TS decreases the decline in accuracy due to updating of LD between markers and QTL, but nonetheless accuracy continues to decline across breeding cycles. Furthermore, it was found that the prediction accuracy was slightly higher when the TS contained only the most recent data, whereas adding the best individuals from each cycle in long-term breeding schemes resulted in the highest genetic gain (Neyhart et al. 2017). Our results revealed that updating methods had a similar genetic variance decreasing across breeding cycles, with the lowest value for $\mathrm{TS}_{\mathrm{GPO}}$ in the tenth breeding cycle.

All updating methods avoided an abrupt decline in prediction accuracy across breeding cycles, which corroborates previous studies (Neyhart et al. 2017; Muleta et al. 2019). In the first cycle, TSC $_{0}$ accuracy presented the highest accuracy (0.69), which can be explained from the TS size effect (Müller et al. 2017; Zhang et al. 2017; Norman et al. 2018). However, over breeding cycles, $\mathrm{TSC}_{0}$ accuracy presented the highest decline displaying negative values from the eighth cycle. $\mathrm{TSC}_{0}$ scenario did not update the training set, and selection candidate genotypes were selected only using the initial marker effects. in accuracy decline (Lorenz and Smith 2015).

When considering the maintenance of accuracy and genetic gain across breeding cycles, a balance needs to be maintained between the genetic relationship between the TS and selection candidate genotypes and accurate estimates of LD between markers and QTL. However, in long-term recurrent schemes, new recombination events occur at each breeding cycle, which causes a breakdown of LD between markers and QTL, consequently decreasing accuracy (Jannink 2010; Müller et al. 2017). Investigating the marker density effect on maize long-term breeding schemes, DoVale et al. (2021) pointed that high-density marker panels reduce an abrupt decline of accuracy across breeding cycles and, consequently, they can deliver higher genetic gain in long-term genomic recurrent schemes. The lower accuracy of low-densities marker panels in our longterm breeding schemes employing GS may be explained by a lower probability of LD between the marker and the QTL when there are fewer markers, which results in a smaller fraction of explained genetic variation (Solberg et al. 2008). Hence, highdensity marker panels could help to increase the probability of finding markers in LD with the same QTL across different cycles (Daetwyler et al. 2010).

This study used a SNP chip containing 1,000 markers to simulate the SNP-panel optimized for the IRRI irrigated breeding program (Arbelaez et al. 2019). Low-density SNP panels are attractive for GS due to the lower price per sample and have been explored by several groups (Vallejo et al., 2018; Arbelaez et al., 2019; Al-Tobasei et al., 2021). In this context, 
408 a lower decay than other studies (Neyhart et al. 2017; Muleta et al. 2019), even using a low-density marker set. Updating the

409 TS promoted more accurate estimates of LD between markers and QTL, since, in long-term breeding schemes, recombination

410 between marker and QTL causes an LD decrease, whereas selection and drift act to generate new LD or tighten the LD

411 between closely linked loci (Hill and Robertson 1968; Habier et al. 2007; Lorenz et al. 2011). This outcome is crucial as SNP

412 chips with few markers may permit breeding programs with limited resources to use GS in their pipeline. However, it seems

413 to be not so essential consider previous cycles to estimate the LD patterns between markers and QTL since $\mathrm{TS}_{\mathrm{ALL}}$ and $\mathrm{TS}_{\mathrm{GPO}}$

414 obtained as similar to those obtained via $\mathrm{TSC}_{\mathrm{N}}$, which counts only the last breeding cycle. However, as described earlier, we

415 considered $\mathrm{TS}_{\mathrm{ALL}}$ a straightforward and stable method. Furthermore, it might buffer and keep the accuracies at good levels in

416 cases of missing a year of trials.

417

418 Conclusion

419

420

In long-term breeding schemes employing genomic selection may deliver higher genetic gain over breeding cycles and over a given period. However, it is crucial to determine where and when to implement the genomic selection since GS 422 can produce lower genetic gain than phenotypic selection, depending on the strategy. Applying genomic selection in an $F_{2}$ 423 progeny followed by a phenotypic selection of new parentals in $\mathrm{F}_{4}$ produced the highest genetic gain across breeding cycles. 424 Moreover, updating the training set allowed better maintenance of accuracy selection over recurrent breeding cycles. Adding 425 a new and proper amount of information every cycle into the training set allows re-estimation of the marker's effects. In other 426 words, updating the LD between markers and underlying QTL guarantees the highest genetic gain over recurrent selection 427 cycles. 


\section{References}

431

432

Al-Tobasei, R., Ali, A., Garcia, A.L.S., Lourenco, D., Leeds, T., Salem, M. (2021). Genomic predictions for fillet yield and firmness in rainbow trout using reduced-density SNP panels. BMC Genomics 22:92. https://doi.org/10.1186/s12864021-07404-9.Arbelaez JD, Dwiyanti MS, Tandayu E, et al (2019) 1k-RiCA ( 1K-Rice Custom Amplicon ) a novel genotyping amplicon-based SNP assay for genetics and breeding applications in rice.

Bassi FM, Bentley AR, Charmet G, et al (2016) Plant Science Breeding schemes for the implementation of genomic selection in. Plant Sci 242:23-36. doi: 10.1016/j.plantsci.2015.08.021

Beyene Y, Semagn K, Mugo S, et al (2015) Genetic gains in grain yield through genomic selection in eight bi-parental maize populations under drought stress. Crop Sci 55:154-163. doi: 10.2135/cropsci2014.07.0460

Brown J, Caligari P, Campos H (2014) Plant Breeding, 2ed edn. Wiley-Blackwell

Cericola F, Jahoor A, Orabi J, et al (2017) Optimizing training population size and genotyping strategy for genomic prediction using association study results and pedigree information. a case of study in advanced wheat breeding lines. PLoS One 12:1-20. doi: 10.1371/journal.pone.0169606

Chen GK, Marjoram P, Wall JD (2009) Fast and flexible simulation of DNA sequence data. Genome Res 19:136-142. doi: 10.1101/gr.083634.108

Cobb JN, Juma RU, Biswas PS, et al (2019) Enhancing the rate of genetic gain in public-sector plant breeding programs: lessons from the breeder's equation. Theor Appl Genet 132:627-645. doi: 10.1007/s00122-019-03317-0

Collard BCY, Beredo JC, Lenaerts B, et al (2017) Revisiting rice breeding methods-evaluating the use of rapid generation advance (RGA) for routine rice breeding. Plant Prod Sci 20:337-352. doi: 10.1080/1343943X.2017.1391705

Collard BCY, Gregorio GB, Thomson MJ, et al (2019) Transforming Rice Breeding: Re-Designing the Irrigated Breeding Pipeline at the International Rice Research Institute (IRRI). Crop Breeding, Genet Genomics 1-19. doi: $10.20900 / \operatorname{cbgg} 20190008$

Combs E, Bernardo R (2013) Accuracy of Genomewide Selection for Different Traits with Constant Population Size, Heritability, and Number of Markers. Plant Genome 6:0. doi: 10.3835/plantgenome2012.11.0030

Crossa J, Pérez-Rodríguez P, Cuevas J, et al (2017) Genomic Selection in Plant Breeding: Methods, Models, and Perspectives. Trends Plant Sci 22:961-975. doi: 10.1016/j.tplants.2017.08.011

Daetwyler HD, Hickey JM, Henshall JM, et al (2010) Accuracy of estimated genomic breeding values for wool and meat 
traits in a multi-breed sheep population. Anim Prod Sci 50:1004-1010.

459 DoVale JC, Carvalho HF, Sabadin F, Fritsche- R (2021) Reduction of genotyping marker density for genomic selection is 460 not an affordable approach to long-term breeding in cross-pollinated crops.

461 Endelman JB (2011) Ridge Regression and Other Kernels for Genomic Selection with R Package rrBLUP. Plant Genome $462 \quad$ 4:250-255. doi: 10.3835/plantgenome2011.08.0024

463 Falconer DS, Mackay TFC (1996) Introduction to Quantitative Genetics. Pearson

464 Garner JB, Douglas ML, Williams SRO, et al (2016) Genomic selection improves heat tolerance in dairy cattle. Sci Rep 465 6:1-9. doi: $10.1038 /$ srep34114

466 Gaynor RC, Gorjanc G, Bentley AR, et al (2017) A two-part strategy for using genomic selection to develop inbred lines. 467 Crop Sci 57:2372-2386. doi: 10.2135/cropsci2016.09.0742

468 Gaynor RC, Gorjanc G, Hickey JM (2021) AlphaSimR: an R package for breeding program simulations. G3 $469 \quad$ Genes|Genomes|Genetics. doi: 10.1093/g3journal/jkaa017

470 Gilmour AR (2007) Mixed model regression mapping for QTL detection in experimental crosses. 51:3749-3764. doi: 10.1016/j.csda.2006.12.031

472 Goddard M (2009) Genomic selection : prediction of accuracy and maximisation of long term response. 245-257. doi: 10.1007/s10709-008-9308-0

Gorjanc G, Gaynor RC, Hickey JM (2018) Optimal cross selection for long-term genetic gain in two-part programs with rapid recurrent genomic selection. Theor Appl Genet 131:1953-1966. doi: 10.1007/s00122-018-3125-3

Gorjanc G, Gaynor RC, Hickey JM, et al Optimal cross selection for long-term genetic gain in a two- part genomic selection strategy. 1-9.

Guo Z, Tucker DM, Basten CJ, et al (2014) The impact of population structure on genomic prediction in stratified populations. Theor Appl Genet 127:749-762. doi: 10.1007/s00122-013-2255-x

Habier D, Fernando RL, Dekkers JCM (2007) The impact of genetic relationship information on genome-assisted breeding values. Genetics 177:2389-2397. doi: 10.1534/genetics.107.081190

Hartl DL, Clark AG (2006) Principles of Population Genetics. Sinauer Associates Inc.

He S, Schulthess AW, Mirdita V, et al (2016) Genomic selection in a commercial winter wheat population. Theor Appl Genet 129:641-651. doi: 10.1007/s00122-015-2655-1

Heffner EL, Jannink J-L, Sorrells ME (2011a) Genomic Selection Accuracy using Multifamily Prediction Models in a 
Heffner EL, Jannink JL, Iwata H, et al (2011b) Genomic selection accuracy for grain quality traits in biparental wheat populations. Crop Sci 51:2597-2606. doi: 10.2135/cropsci2011.05.0253

Hill WG, Robertson A (1968) Linkage disequilibrium in finite populations. Theor Appl Genet 38:226-231. doi: 10.1007/BF01245622

Jannink JL (2010) Dynamics of long-term genomic selection. Genet Sel Evol 42:1-11. doi: 10.1186/1297-9686-42-35

Jannink JL, Lorenz AJ, Iwata H (2010) Genomic selection in plant breeding: From theory to practice. Briefings Funct Genomics Proteomics 9:166-177. doi: 10.1093/bfgp/elq001

Li L, Lu K, Chen Z, et al (2008) Dominance, overdominance and epistasis condition the heterosis in two heterotic rice hybrids. Genetics 180:1725-1742. doi: 10.1534/genetics.108.091942

Lorenz AJ (2013) Resource Allocation for Maximizing Prediction Accuracy and Genetic Gain of Genomic Selection in Plant Breeding: A Simulation Experiment. G3\&amp;\#58; Genes|Genomes|Genetics 3:481-491. doi: $10.1534 / \mathrm{g} 3.112 .004911$

Lorenz AJ, Chao S, Asoro FG, et al (2011) Genomic Selection in Plant Breeding. Knowledge and Prospects., 1st edn. Elsevier Inc.

Lorenz AJ, Smith KP (2015) Adding genetically distant individuals to training populations reduces genomic prediction accuracy in Barley. Crop Sci 55:2657-2667. doi: 10.2135/cropsci2014.12.0827

Lorenz AJ, Smith KP, Jannink J (2012) Potential and Optimization of Genomic Selection for Fusarium Head Blight Resistance in Six-Row Barley. 1609-1621. doi: 10.2135/cropsci2011.09.0503

Marulanda JJ, Mi X, Melchinger AE, et al (2016) Optimum breeding strategies using genomic selection for hybrid breeding in wheat, maize, rye, barley, rice and triticale. Theor Appl Genet 129:1901-1913. doi: 10.1007/s00122-016-2748-5

Mendonça LDF, Fritsche-neto R, Galli G, Malone G (2020) Genomic prediction enables early but low-intensity selection in soybean segregating progenies. doi: 10.1002/csc2.20072

Meuwissen THE, Hayes BJ, Goddard ME (2001) Prediction of total genetic value using genome-wide dense marker maps. Genetics 157:1819-1829. doi: 11290733

Michel S, Ametz C, Gungor H, et al (2016) Genomic selection across multiple breeding cycles in applied bread wheat breeding. Theor Appl Genet 129:1179-1189. doi: 10.1007/s00122-016-2694-2

Muleta KT, Pressoir G, Morris GP (2019) Optimizing genomic selection for a sorghum breeding program in Haiti: A simulation study. G3 Genes, Genomes, Genet 9:391-401. doi: 10.1534/g3.118.200932

Müller D, Schopp P, Melchinger AE (2017) Persistency of prediction accuracy and genetic gain in synthetic populations 
517 Neyhart JL, Tiede T, Lorenz AJ, Smith KP (2017) Evaluating methods of updating training data in long-term genomewide $518 \quad$ selection. G3 Genes, Genomes, Genet 7:1499-1510. doi: 10.1534/g3.117.040550

519 Norman A, Taylor J, Edwards J, Kuchel H (2018) Optimising genomic selection in wheat: Effect of marker density, 520 population size and population structure on prediction accuracy. G3 Genes, Genomes, Genet 8:2889-2899. doi: $521 \quad 10.1534 / \mathrm{g} 3.118 .200311$

522 Solberg TR, Sonesson AK, Woolliams JA, Meuwissen THE (2008) Genomic selection using different marker types and 523 densities. J Anim Sci 86:2447-2454. doi: 10.2527/jas.2007-0010

524 Spindel J, Begum H, Akdemir D, et al (2015) Genomic Selection and Association Mapping in Rice (Oryza sativa): Effect 525 of Trait Genetic Architecture, Training Population Composition, Marker Number and Statistical Model on Accuracy 526 of Rice Genomic Selection in Elite, Tropical Rice Breeding Lines. PLoS Genet 11:1-25. doi:

$527 \quad$ 10.1371/journal.pgen.1004982

528 Varshney RK, Roorkiwal M, Sorrells ME (2017) Genomic selection for crop improvement: An introduction. 529 Voss-Fels KP, Cooper M, Hayes BJ (2019) Accelerating crop genetic gains with genomic selection. Theor Appl Genet $530 \quad$ 132:669-686. doi: 10.1007/s00122-018-3270-8

531 Wolc A, Zhao HH, Arango J, et al (2015) Response and inbreeding from a genomic selection experiment in layer chickens. 532 Genet Sel Evol 47:1-12. doi: 10.1186/s12711-015-0133-5

533 Zhang A, Wang H, Beyene Y, et al (2017) Effect of trait heritability, training population size and marker density on 534 genomic prediction accuracy estimation in 22 bi-parental tropical maize populations. Front Plant Sci 8:1-12. doi: $535 \quad 10.3389 /$ fpls.2017.01916

536 Zhong S, Dekkers JCM, Fernando RL, Jannink J (2009) Factors Affecting Accuracy From Genomic Selection in 537 Populations Derived From Multiple Inbred Lines: A Barley Case Study. doi: 10.1534/genetics.108.098277 
Figures

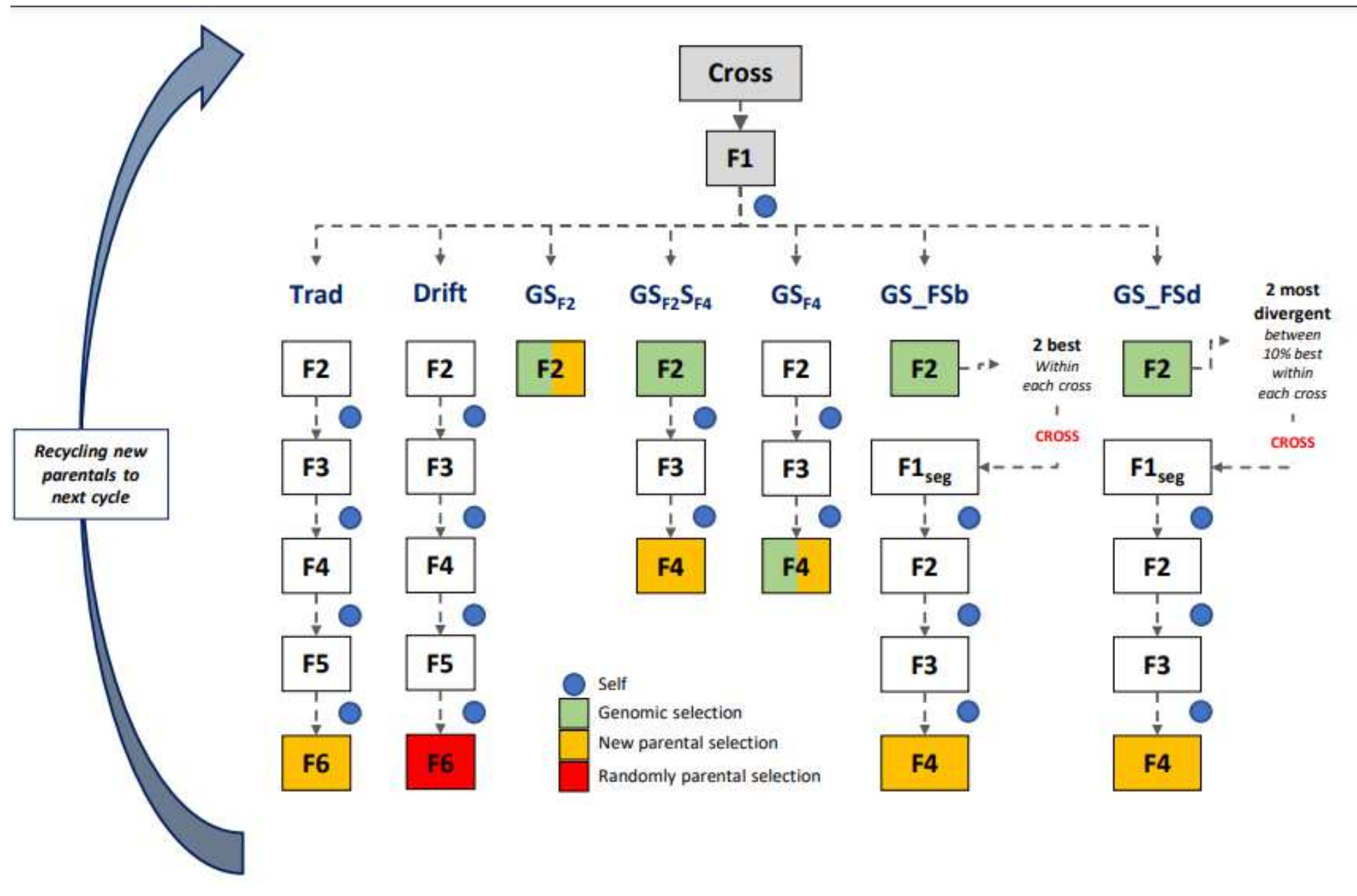

Figure 1

Scheme of the first cycle of the long-term breeding schemes. In the final of each scheme, a parental group was selected for recycling and compound the next cycle that works independently 

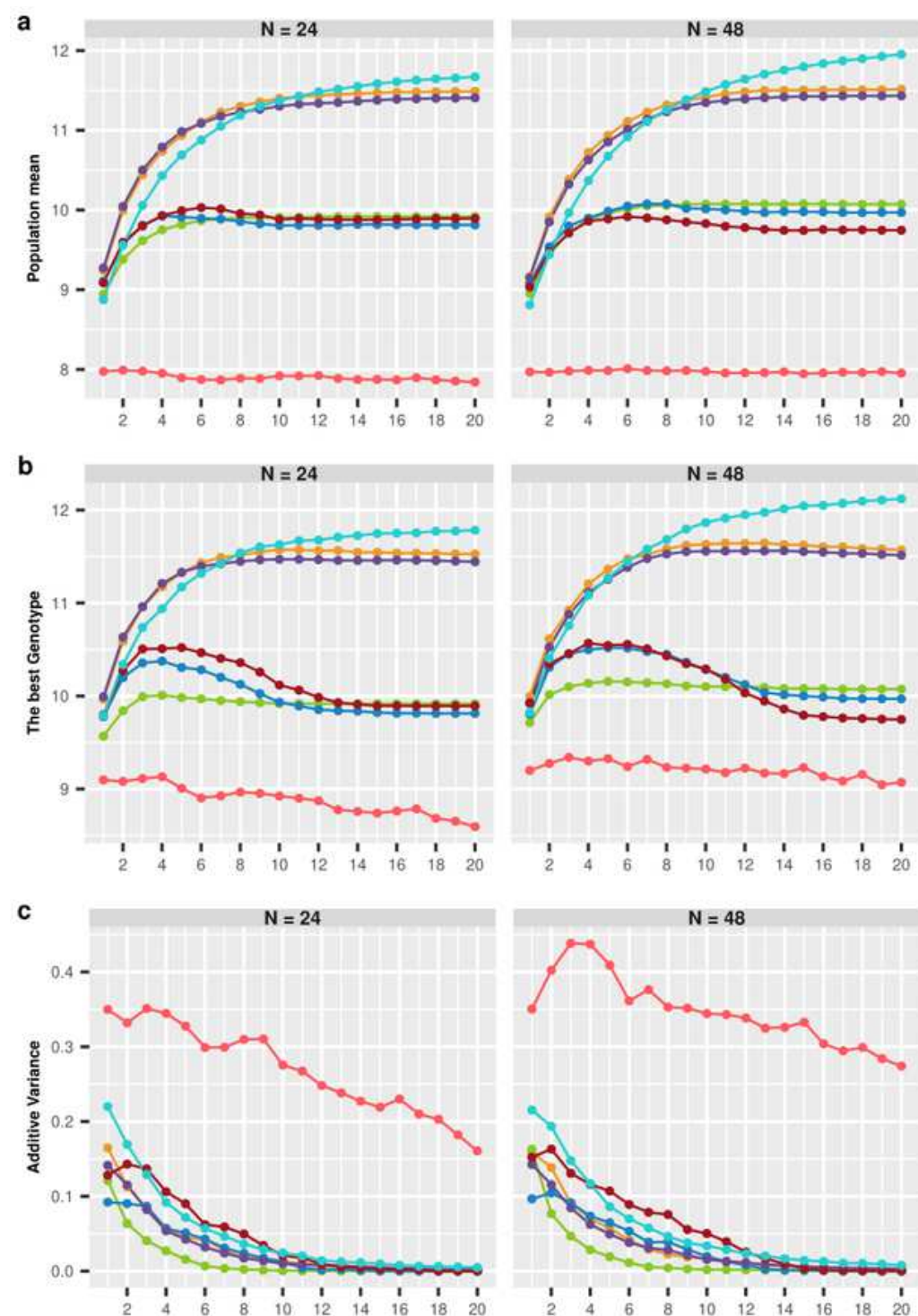

method

$\because$ DRIFT

$\because$ GS_FSb

$\rightarrow$ GS FSd
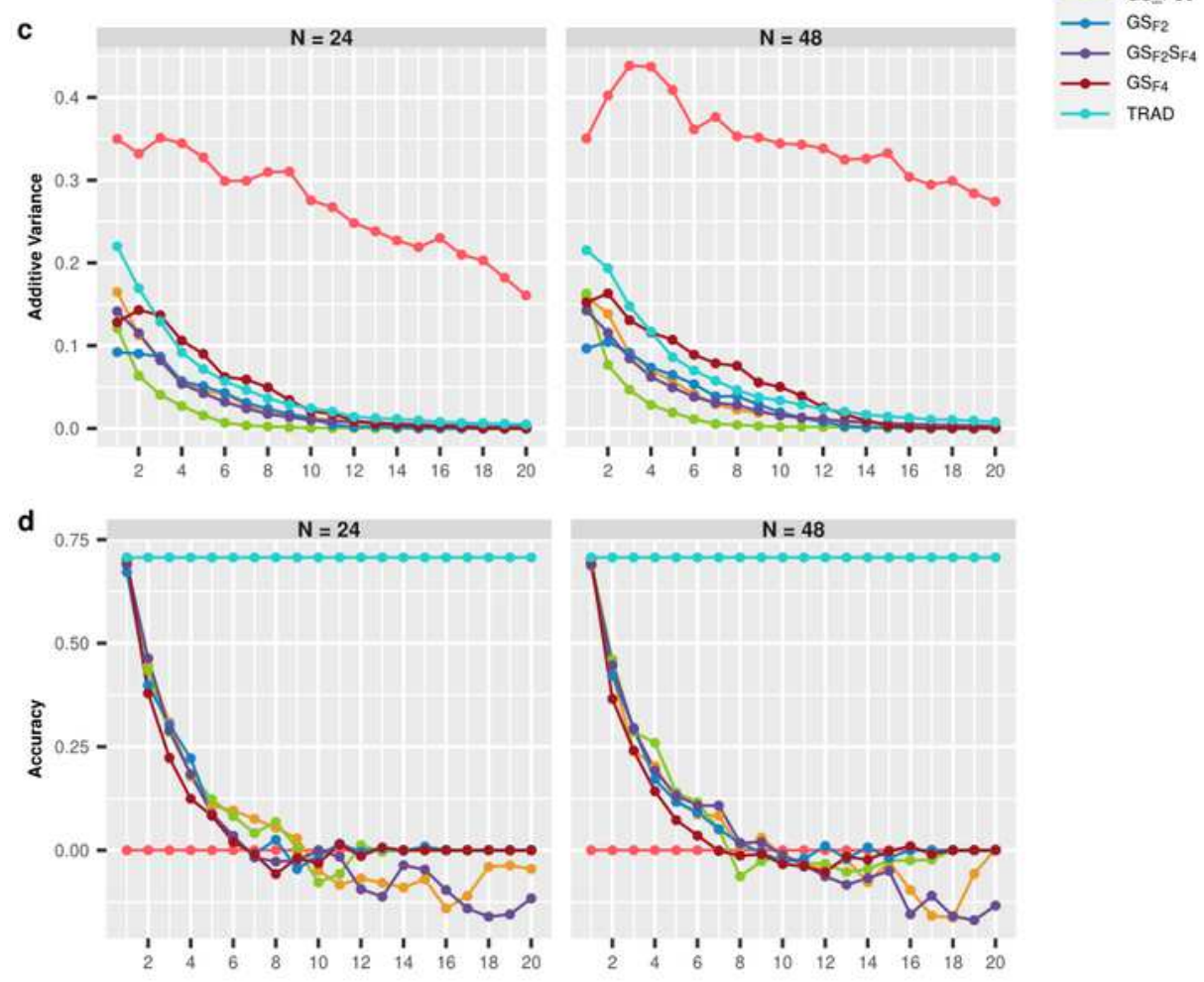

cycles

Figure 2

Population mean (a), the best genotype (b), additive variance (c), and accuracy of selection (d) over 20 long-term breeding schemes using two sizes of parental lines $(\mathrm{N}=24$ and $\mathrm{N}=48)$. Each colored line represents a breeding scheme. 
$\mathrm{Ne}$ 24 48

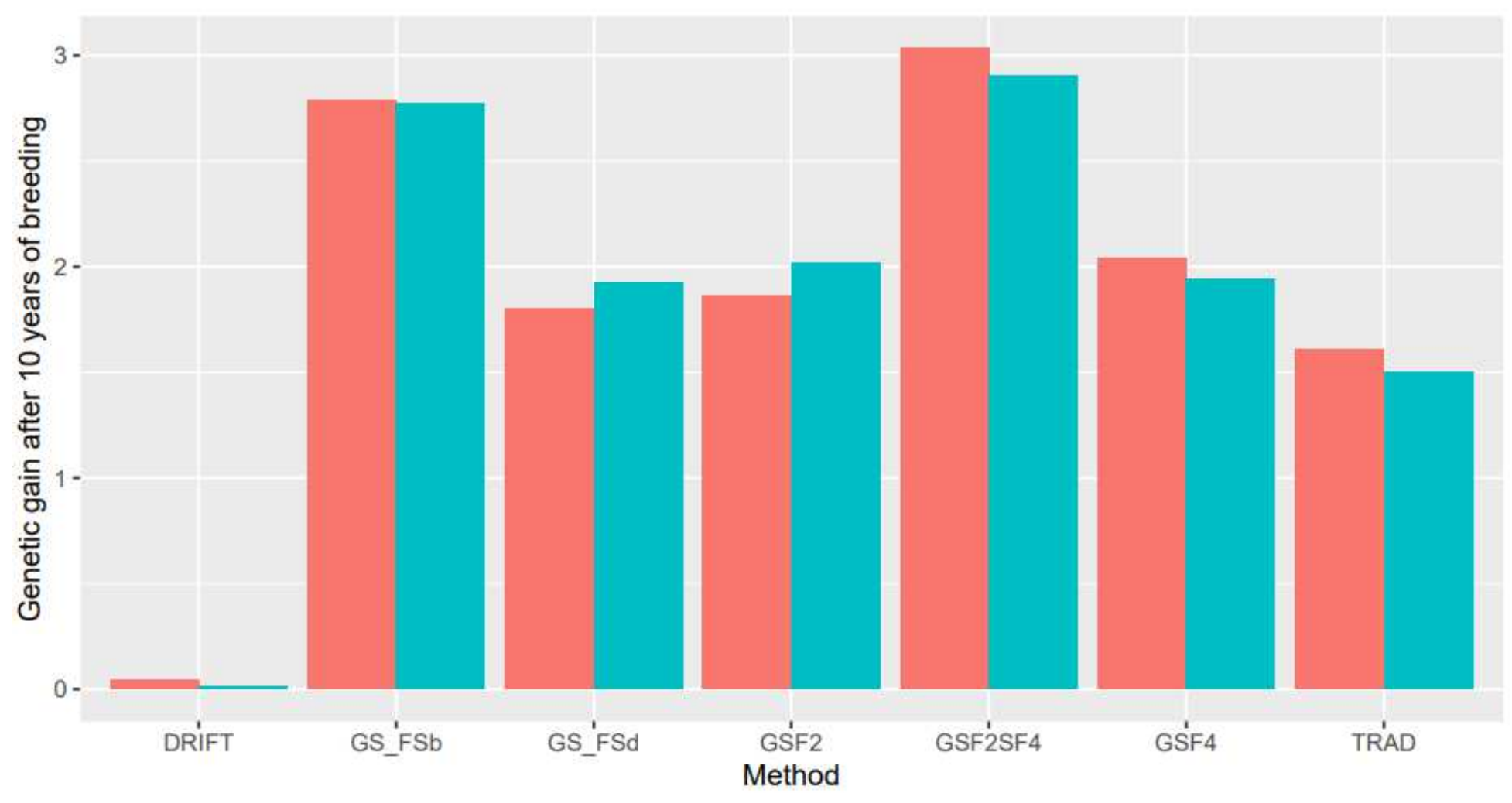

Figure 3

Genetic gains after ten years of breeding using different selection schemes and two sizes of parental lines $(\mathrm{N}=24$ and $\mathrm{N}=48$. 


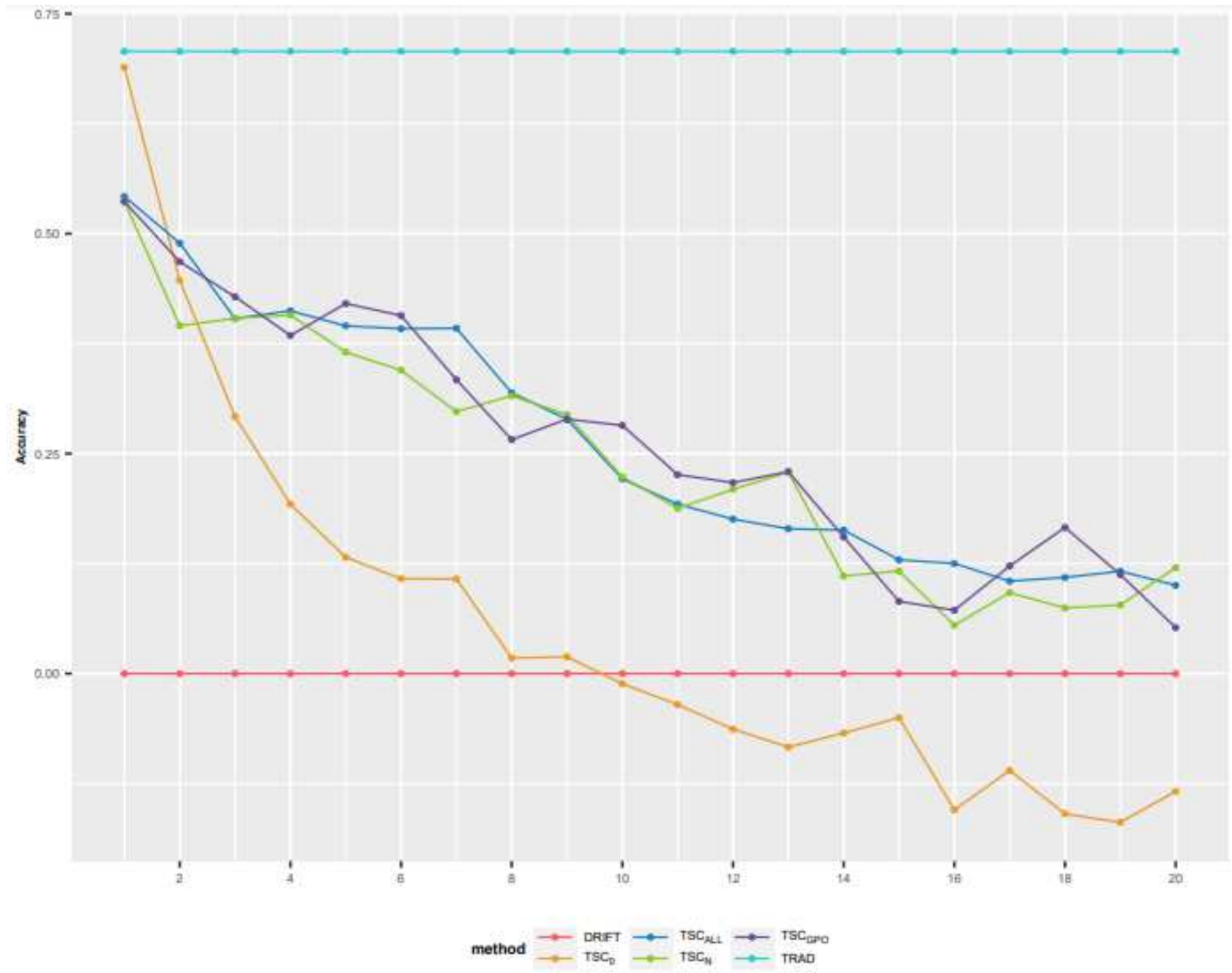

Figure 4

Accuracy of selection over 20 breeding cycles via GSF2SF4 scheme considering 48 parental lines using different methods to update the training set. Each colored line represents an updating training set method. 


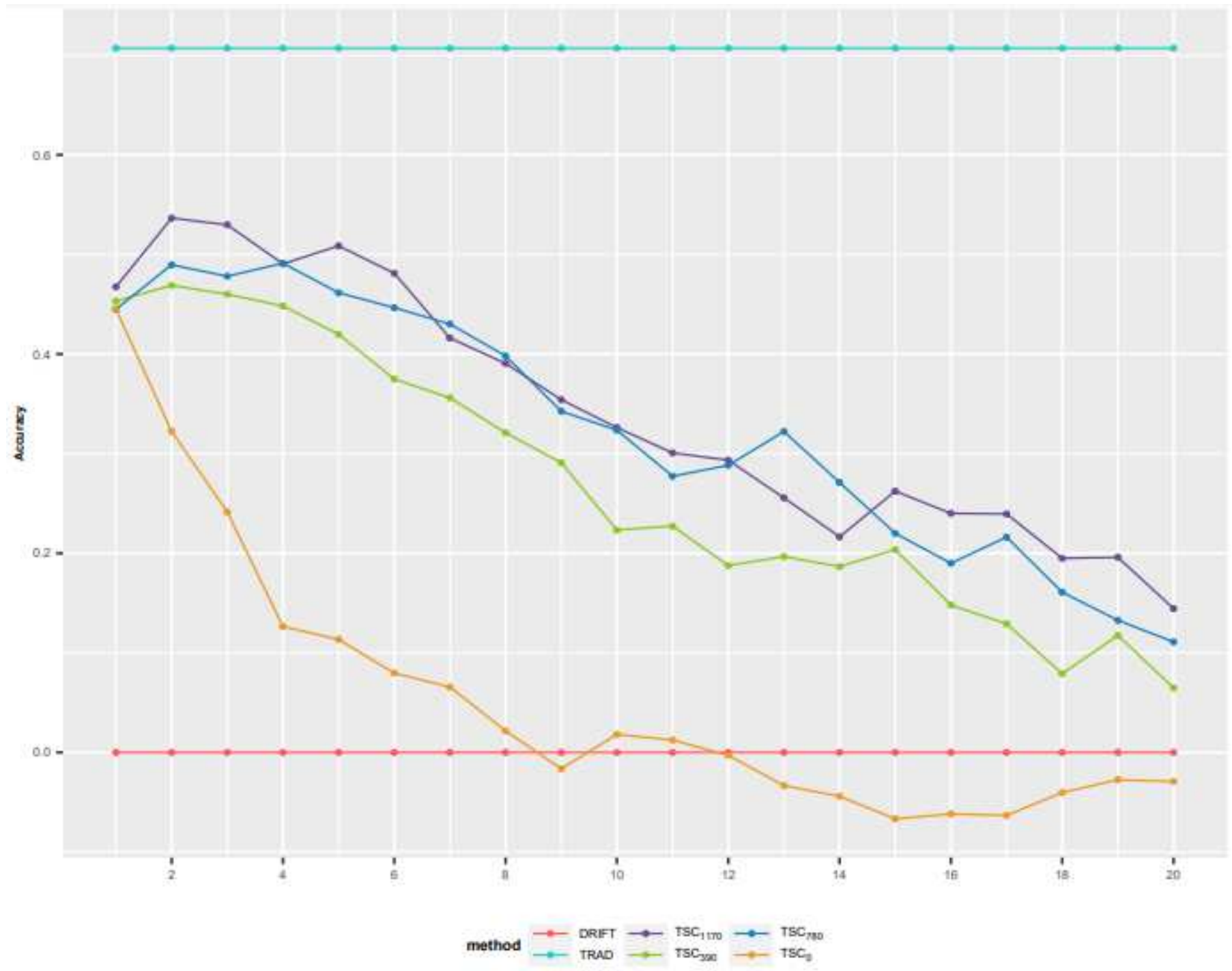

Figure 5

Accuracy of selection over 20 breeding cycles via GSF2SF4 scheme considering 48 parental lines, the TSALL method, and different sample sizes to update every cycle's training set. Each colored line represents an updating training sample size.

\section{Supplementary Files}

This is a list of supplementary files associated with this preprint. Click to download.

- IRRIBPGSTSforeach.r

- IRRIGSTSsizesforeach.r 